Chirurg 2010 $\cdot 81: 689-690$

DOI 10.1007/s00104-010-1951-1

Online publiziert: 15. Juli 2010

(c) Springer-Verlag 2010

\section{J. Jähne}

Zentrum Chirurgie, Klinik für Allgemein- und Visceralchirurgie, Schwerpunkt für endokrine und onkologische Chirurgie, Diakoniekrankenhaus Henriettenstiftung Hannover gGmbH, Diakonische Dienste Hannover

\title{
Management in der Chirurgie
}

\section{Herausforderungen heutiger Führungspositionen}

Sie als Leser der Zeitschrift Der Chirurg werden sich vielleicht wundern, dass Schriftleitung und Herausgeber das Thema „Management in der Chirurgie“ als ein Leitthema für das Jahr 2010 ausgewählt haben. Auch in der Herausgebersitzung zur Festlegung der Leitthemen wurde durchaus kontrovers diskutiert, ob sich eine wissenschaftliche Fachzeitschrift dieser Thematik annehmen soll. In Anbetracht der zunehmenden ökonomischen Herausforderungen in der Chirurgie überwog die Überzeugung, mit diesem Leitthema einen Beitrag zu einer differenzierten Darstellung und vielleicht auch ein wenig Überzeugungsarbeit zu leisten.

Auch wenn eigentlich allen Chirurgen die Rahmenbedingungen ihrer ärztlichen Tätigkeit klar sein sollten - demographischer Wandel, technischer Fortschritt, Fachkräftemangel, finanzielle Limitationen, um nur einige zu nennen - stößt das Thema Management, oftmals reduziert auf den Begriff „Ökonomisierung“, meist mit dem Verweis auf das Primat einer optimalen Patientenversorgung auf eine breite Ablehnung. Dies ist umso erstaunlicher - und auch nicht verständlich -, da sich zum einen ein gutes Klinikmanagement positiv auf die Patientenbehandlung auswirkt und zum anderen wir alle in unserem täglichen Leben mit dem Anspruch an ein zufriedenstellendes Gelingen mit begrenzten Ressourcen umgehen und im persönlichen Miteinander immer auch unsere soziale, emotionale und moralische Intelligenz einfließen lassen müssen. Das Management einer Klinik betrifft eben nicht nur die monetären Aspekte, sondern beinhaltet auch so unterschiedliche Facetten wie Führung, Mitarbeitergewinnung und -motivation, Marketing sowie Strategieentwicklung.

\section{> Klinikmanagement beinhaltet monetäre Aspekte, Mitarbeiterführung und Strategieentwicklung}

Unstrittig ist, dass für eine erfolgreiche chirurgische Tätigkeit das rein medizinisch-chirurgische Patientenmanagement von der Indikation zum Eingriff und der Operation selbst bis hin zur periund postoperativen Nachsorge auf der Basis gesicherter chirurgisch-wissenschaftlicher Erkenntnisse eine Conditio sine quo non darstellt, um eine optimale $\mathrm{Pa}$ tientenversorgung auf einem hohen Qualitätsniveau zu gewährleisten. Dies ist die Kernaufgabe chirurgischer Tätigkeit, und sie wird es immer bleiben! Wie Rainer Arbogast in seinem Beitrag sehr zutreffend formuliert, ist es die medizinische Kompetenz, auf der sich die Akzeptanz einer Chirurgenpersönlichkeit gründet. Darin sind sich trotz unterschiedlicher Auffassungen über das Ausmaß der Managementzusatzqualifikationen alle Autoren zu diesem Leitthema einig. Mit der chirurgischen Fachkompetenz sind die Übernahme und Weitergabe von Verantwortung ebenso wie das „Beispiel-Geben“ eng assoziiert. Viele dieser Fähigkeiten können nicht erlernt werden, sondern sind mit der (authentischen) Persönlichkeit eines jeden Chirurgen mehr oder weniger verbunden. Und nicht jeder Chirurg hat das, was Arbogast mit Charisma umschreibt - die natürliche Fähigkeit zur Führung nicht nur der Patienten und Mitarbeiter, sondern auch die Verantwortung für die Klinik, deren Existenz und $\mathrm{Zu}$ kunftssicherheit. Auch aus der Perspektive des langjährig erfolgreichen Chefarztes wird deutlich, dass mehr als nur die optimale Patientenversorgung erforderlich ist, um heute den gestiegenen Führungsaufgaben gerecht zu werden.

Hier scheiden sich die Geister! Wie viel Managementkompetenz muss eine chirurgischeFührungspersönlichkeithabenundvor allem - wie erwirbt sie diese Kenntnisse? In Anbetracht der sich dramatisch veränderten Finanzierungssysteme, der begrenzten finanziellen Ressourcen, der zunehmenden Konkurrenzsituation der Krankenhäuser, des demographischen Wandels und der Fortschritte der medizinischen Technik sind vielfältige Instrumente eines effektiven und effizienten Managements notwendig, um Krankenhäuser aus ihrem bisherigen Dasein von Versorgungsanstalten zu leistungsstarken Dienstleistungsunternehmen - denn nichts anderes ist eine erfolgreich operierende Klinik - zu entwickeln. Auf der einen Seite stehen die Protagonisten für ein zusätzliches berufsbegleitendes Studium zum Erwerb von Managementkenntnis- 
sen, auf der anderen Seite die Verfechter einer gezielten Weiterqualifikation in spezifischen Bereichen. Wegen der Komplexität einer erfolgreichen Klinikführung und der Planung individueller Karrierewege sind MBA (Master-of-Business-Administration)-Studiengänge durchaus geeignet, diese Kenntnisse zu erwerben. Bork und Mitarbeiter zeigen den Stellenwert solcher Studiengänge absolut nachvollziehbar auf, wobei natürlich nicht unberücksichtig bleiben darf, dass die Auswahl eines geeigneten Studiengangs aufgrund der Fülle der bestehenden Angebote nicht einfach ist, zumal sich hier auch ein Markt entwickelt hat, in dem sich die unterschiedlichsten Anbieter mit einem durchaus divergierenden Angebot und Niveau tummeln.

In jedem Fall jedoch führt ein berufsbegleitendes MBA-Studium zu einem übergeordneten Denken in größeren $\mathrm{Zu}$ sammenhängen und erlaubt, aus der Sicht der Vogelperspektive die Betroffenheitskrise des Arztes über die sich wandelnden ökonomischen Begleitumstände $\mathrm{zu}$ überwinden. Dabei ist durchaus zu realisieren, dass Ärzte mit Managementwissen in den gegenwärtigen Organisationsstrukturen der Krankenhäuser nicht unbedingt akzeptiert sind. Der Ausspruch von E. Münch: „Sorgen Sie dafür, dass der Faden richtig abgeschnitten wird, den Rest machen wir. "1 mag hier stellvertretend für solche Sichtweisen sein, die sich aber offensichtlich auch bei privaten Trägern geändert haben. Richter und Mitarbeiter zeigen mit ihrer Darstellung des Anforderungsprofils an den chirurgischen Chefarzt, dass neben der chirurgischen Fachkompetenz eben doch auch entsprechendes betriebswirtschaftliches Denken und Handeln notwendig sind, stehen aber einem qualifizierenden zusätzlichen MBA-Studium eher skeptisch gegenüber.

In diesem Kontext sehen Tecklenburg und Liebeneiner daher den MBA als zu unspezifisch und undefiniert und plädieren für gezielte Weiterbildungsmaßnahmen, um spezifische Defizite abzubauen. Bei einem MBA-Studium geht es allerdings nicht darum, alles abzudecken und beim Management einer Klinik alles

1 Erwiderung auf einen Vortrag von M. Büchler beim Symposium Quo vadis 2005. selbst zu machen. Vielmehr ist es hilfreich, mit dem erworbenen Wissen die von anderen erarbeitete Information kritisch hinterfragen zu können. Natürlich muss kein Arzt eine Deckungsbeitragsrechnung selbst erstellen, aber er muss wissen, wie sie zustande kommt. Er muss auch wissen, wie das Marketing seiner Abteilung funktioniert, und er muss ein Gespür dafür bekommen, welche Strategie für seine Klinik unter Beachtung der strukturellen und personellen Ressourcen möglich ist. Darüber hinaus sind auch makroökonomische Kenntnisse durchaus hilfreich, um das Funktionieren des Marktes „Gesundheitswirtschaft“ zu verstehen. Hinzu kommt die große Frage, ob es durch spezifische Seminare wirklich gelingt, eigene Schwächen abzubauen. Oftmals ist es besser, seine Stärken zu stärken und die Schwächen zu akzeptieren! Und natürlich enthebt ein MBA-Studium den Arzt nicht von der Bereitschaft zum lebenslangen Lernen nicht nur auf chirurgischem Terrain.

\section{(7) Neben der chirurgischen Fachkompetenz sind betriebs- wirtschaftliches Denken und Handeln notwendig}

Dabei werden gerade die von Tecklenburg und Liebeneiner favorisierten (Führungs-) Seminare immer noch kritisch betracht, wie Petra Schubert ausführt. In erster Linie sollen sie dazu dienen, sich als Führungskraft immer wieder zu hinterfragen. Obwohl Chirurgen eigentlich aufgrund ihrer Tätigkeit zur Selbstkritik prädestiniert sein müssten, fehlt vielen diese Fähigkeit bei der Betrachtung ihrer nichtmedizinischen Kompetenzen. Hier besteht sicher noch ein großer Nachholbedarf, da chirurgische Allmachtsphantasien den Realitäten einer modernen Krankenhausführung geradezu diametral entgegenstehen. Es darf allerdings auch nicht vergessen werden, dass eine Veränderbarkeit der persönlichen Kompetenz der Führungskraft wesentlich schwieriger gelingt als eine Optimierung der Verhaltensund Methodenkompetenz, somit also die Teilnahme an solchen Seminaren durchaus sinnvoll sein kann.

Selbst wenn aber das Hinterfragen der eigenen Führung gelingt und vielfäl- tige nichtchirurgische Managementfähigkeiten erworben werden, scheitern dennoch viele Chefärzte in ihrer neuen Position und Funktion, weil von den Klinikträgern oftmals Situationen geschaffen werden, die von Beginn an den Konflikt programmieren. Heberer und Hüttl zeigen deutlich, dass viele Klinikleitungen zu sehr in Zahlen denken, ohne die Situation analytisch zu erfassen und dann gezielt ärztliches Personal zu suchen. Auch wird offensichtlich, dass jeder, der eine leitende Position anstrebt, sich von der zukünftigen Führungsaufgabe nicht blenden lassen sollte, sondern durchaus in der Lage sein muss, eine noch so attraktive Position abzulehnen, wenn die Voraussetzungen nach seiner eigenen kritischen Analyse nicht stimmen.

Allen Beiträgen ist die Erkenntnis gemeinsam, dass die Zukunft der Krankenhäuser nur dann erfolgreich sein wird, wenn Chirurgen als Manager ihrer jeweiligen Abteilungen und das Management des Krankenhauses vertrauensvoll und eng zusammenarbeiten. Management in der Chirurgie ist - wie im fachlich-chirurgischen - interdisziplinär: Arzt, Geschäftsführer, Controlling, Einkauf, Finanzwesen und Personal sind keine Gegenpole, sondern sich ergänzende und unterstützende Personen und Einrichtungen, die ihre jeweiligen Fähigkeiten zur erfolgreichen Zukunftssicherung der Kliniken bündeln und gemeinsam agieren müssen. Der Arzt und Chirurg als Manager sollte deshalb kein Feindbild sein, sondern eine willkommene Ergänzung zu einer modernen Krankenhaus(betriebs)führung.

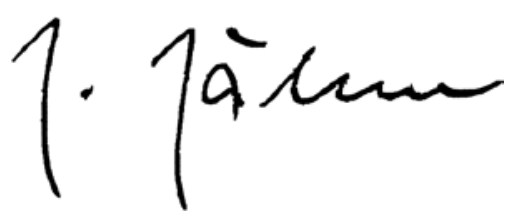

Prof. Dr. J. Jähne, MBA

\section{Korrespondenzadresse \\ Prof. Dr. J. Jähne}

Zentrum Chirurgie, Klinik für Allgemein- und Visceralchirurgie, Schwerpunkt für endokrine und onkologische Chirurgie, Diakoniekrankenhaus Henriettenstiftung Hannover $\mathrm{gGmbH}$, Diakonische Dienste Hannover, Marienstraße 72-90, 30171 Hannover joachim.jaehne@ddh-gruppe.de 